Levy, E. S. (2014). Implementing two treatment approaches to childhood dysarthria.

International Journal of Speech-Language Pathology, 16, 344-354.

http://dx.doi.org/10.3109/17549507.2014.894123

Accepted for publication Feb. 9, 2014

Implementing two treatment approaches to childhood dysarthria

\author{
Erika S. Levy \\ Teachers College, Columbia University
}

Correspondence concerning this article should be addressed to Erika S. Levy, Biobehavioral

Sciences, Teachers College, Columbia University, 525 W $120^{\text {th }}$ St., Box 5, New York, NY 10027

Contact: elevy@tc.columbia.edu

Key words: intelligibility, childhood dysarthria, speech treatment, treatment research

\begin{abstract}
The paucity of evidence and detail in the literature regarding speech treatment for children with dysarthria due to cerebral palsy $(\mathrm{CP})$ renders it difficult for researchers to replicate studies and make further inroads into this area in need of exploration. Furthermore, for speech-language pathologists (SLPs) wishing to follow treatments that the literature indicates have promise, little guidance is available on the details of the treatments that yielded the positive results. The present article details the implementation of two treatment approaches in speech treatment research for children with dysarthria: Speech Systems Intelligibility Treatment (SSIT) and the Lee Silverman Voice Treatment LOUD (LSVT LOUD). Specific strategies, primarily for treatment, but also for outcome measurement and acoustic analysis of dysarthric speech, are described. These techniques are provided for researchers and clinicians to consider implementing in order to advance speech treatment for this population. Recent results from research using these approaches are presented, including findings of acoustic vowel space changes following both speech treatments.
\end{abstract}


Implementing two treatment approaches to childhood dysarthria

This article originated from several observations regarding speech treatment for children with cerebral palsy (CP): First, despite the large number of children with $\mathrm{CP}$ and the oftaccompanying motor speech disorder of dysarthria, childhood dysarthria treatment is an underexplored area of research (Pennington, Miller, \& Robson, 2009). Moreover, within the limited research, few specifics are offered regarding how treatment was performed. Because research often progresses through replication and extension or expansion of previous studies, the paucity of evidence and detail renders it difficult for researchers to replicate and make further inroads into this area in need of exploration. Furthermore, speech-language pathologists (SLPs) working with children with dysarthria have sparse evidence on which to base their treatment. For SLPs wishing to follow as closely as possible treatments that the literature indicates have promise, little guidance is available on the details of the treatments that yielded the positive results.

In addition, treatments modeled on successful treatment for adults with dysarthria must recognize that speech treatment for adults involves rehabilitating a speech system that was once intact, whereas treating children involves treating a developing motor control, speech sound, cognitive, and linguistic system (Green, Moore, \& Reilly, 2002). Unlike many adults who have acquired dysarthria after childhood, children with dysarthria due to CP are likely to have phonological and language deficits, along with their speech (and sometimes cognitive) deficits, and atypical development in one domain may affect the other (Goffman, 2004; Hustad, Gorton, \& Lee, 2010; Smith \& Goffman, 2004; Strand, 1992). Thus, the nature of and mechanisms of change in childhood dysarthria will likely differ from those in adult dysarthria (Kent, 2000).

The present article summarizes recent findings, including new acoustical analyses, from research using two approaches to treatment of childhood dysarthria due to cerebral palsy, namely systems-based approaches (e.g., Hodge \& Wellman, 1999; Levy, Ramig, \& Camarata, 2012; Pennington Miller, Robson, \& Steen, 2010; Pennington, Roelant, Thompson, Robson, Steen, \& Miller, 2013; Pennington, Smallman, \& Farrier, 2006; Strand, 1995) and the Lee Silverman Voice Treatment LOUD (LSVT LOUD) (Fox \& Boliek, 2012; Levy et al., 2012). Specific techniques for speech treatment and outcome measurement are offered for researchers and clinicians to consider implementing in order to advance speech treatment for this population. Speech Systems Intelligibility Treatment and LSVT LOUD

Within the limited literature on speech treatment for children with dysarthria due to $\mathrm{CP}$, systems-based approaches and LSVT LOUD have shown promise for improving speech function. The Speech Systems Intelligibility Treatment (SSIT) implemented in the present study is a systems-based approach that draws upon the literature on systems-based treatments for dysarthria (e.g., Hodge \& Wellman, 1999; Pennington et al., 2006, 2010, 2013; Strand, 1995) and follows motor learning principles (Strand, 1992). Improved function and coordination of the subsystems of speech (respiration, phonation, resonance, and articulation) are targeted based on the needs of each child. Studies by Pennington and her colleagues (2006; 2010; 2013) have implemented a systems-based protocol that focuses on stabilizing respiratory and phonatory control and effort, and adjusting phrase length, and speech rate or syllables per breath. Following such treatment, Pennington et al. (2010) found that older children with moderate to severe (spastic, dyskinetic or mixed) dysarthria (ages 12-18) produced 12-16\% more intelligible single words (as measured by selection of target, given 10 phonetically similar words on the Children's Speech Intelligibility Measures [Wilcox \& Morris, 1999]) and connected speech (as measured by number of words heard correctly). Similar findings were revealed at the word level in Pennington et al. (2006), 
although gains were not made at the sentence level. More recently, Pennington et al. (2013) administered treatment to 15 younger children (ages 5-11) with CP (type: spastic, dyskinetic, ataxic, and Worster Drought syndrome) and moderate to severe dysarthria. Speech intelligibility (to unfamiliar listeners), using similar measures to Pennington et al. (2010), increased 9.3\% for single words and $10.5 \%$ for connected speech. Gains were maintained 12 weeks after treatment.

The second approach discussed here, LSVT LOUD, is adapted from adult dysarthria treatment and uses a single target of healthy vocal loudness. The target "loud" is posited to trigger positive effects distributed across speech production systems (Sapir, Spielman, Ramig, Story, \& Fox, 2007). LSVT LOUD has level 1 evidence indicating efficacy for use with hypokinetic dysarthria in adults due to Parkinson Disease (PD) (Ramig, Sapir, Fox, \& Countryman, 2001). Fox and Boliek (2012) found that when LSVT LOUD was adapted for 5-7 year old children with spastic dysarthria due to $\mathrm{CP}$, adult listeners preferred most of the children's speech characteristics post-treatment over pre-treatment. (See Boliek and Fox, 2014, for further information on effects of LSVT LOUD on childhood dysarthria.) The games and activities used for both treatment approaches were developed for this study in the Speech Production and Perception Lab at Teachers College, Columbia University.

Findings from a study performed in the Speech Production and Perception Lab were reported by Levy et al. (2012). In this study, both SSIT and LSVT LOUD were implemented on children with spastic dysarthria due to cerebral palsy, ages 3.3 to 9.6. Following both treatments, higher articulatory accuracy was found on the Arizona Articulation Proficiency Scale- $3^{\text {rd }}$ Edition (AAPS-3) (Fudala, 2001) for all children treated (mean increase $=13 \mathrm{pts}$ ). Furthermore, blinded listeners judged post-treatment utterances as more intelligible (58\% at word-level, $77 \%$ in spontaneous speech) and preferred (57\% at word-level, 76\% in spontaneous speech) than pretreatment utterances. Speech after LSVT LOUD (Fox \& Boliek, 2012) generally was characterized by a higher sound pressure level (mean increase $=8 \mathrm{~dB}$ ), whereas after SSIT, no increase in sound pressure level was found. In summary, both treatments show encouraging results for improving speech function in children with dysarthria, although post-treatment changes in the subsystems of speech may differ and improvements may vary across linguistic levels and children.

At this early phase of treatment research (Robey, 2004) on childhood dysarthria, it is premature to determine which subpopulations are more likely to benefit from particular approaches. Rather, continuing to examine the feasibility and, ultimately, the effects of treatments is necessary for further developing the treatments (Butler \& Darrah, 2001; Pennington et al., 2009). Clearly-described treatment protocols permit researchers to replicate and expand upon studies systematically and thus interpret responses to treatment without confounds introduced when treatment methods vary substantially from study to study.

In the speech treatment studies at the Speech Production and Perception Lab, children (ages 3 to 13 years thus far) are randomly assigned to each treatment condition. In our efforts to understand the principal treatment approaches reported in the literature, determining the treatment protocols followed in previous studies has been one of our greatest challenges. Given that the focus of most treatment articles is the efficacy or promise of treatments and that page limits restrict the level of detail provided, few specifics are typically offered regarding the treatment techniques and outcome measurement protocols. For example, in systems-based approaches, clarification is often needed regarding precisely what tasks are used and how those tasks can be facilitated and motivated in children with CP. Similarly, adapting LSVT LOUD (Ramig \& Fox, 2010) for pediatric populations often necessitates adjustments of the adult protocol. We emphasize that the specifics provided here regarding procedures used in our treatment research 
are only one interpretation of such treatments - other implementations may also yield promising results.

\section{The specifics of treatment}

Preparing for treatment

In preparing for treatment, we ask parents to help their children generate a list of phrases the children use daily at home or in school. These become the practice phrases for SSIT or functional phrases for LSVT LOUD. Sample phrases include "Who's on the phone?", "Where's the bathroom?", and "Have an amazing day!" In addition, parents are asked for a list of the child's favorite topics of discussion, games, activities, and rewards, which we use for the child's engagement and motivation.

Treatment takes place in a typical therapy room or a laboratory in which distracting toys (and mirrors) are removed from the child's visual field. Whenever possible, we arrange for two student clinicians to attend to the child. The primary clinician treats the child; the other clinician helps motivate the child, logs responses, and assists the primary clinician. In addition, because timing is essential in treatment studies, this second clinician serves as a substitute if the primary clinician is absent. For both treatments, talented students with some related experience are recommended by the clinic and are supervised by the (LSVT LOUD-certified) primary investigator or the primary investigator provides services. According to LSVT Global (2013), students (or SLPs) providing LSVT LOUD must be LSVT LOUD-certified before beginning and should have treated at least three clients before performing the treatment for research purposes. Materials used are appropriate for the child's age, cultural background, and cognitive, linguistic, and physical abilities. Wheelchair access is made possible and activities are adapted for children with motor deficits and those who might fatigue easily.

During treatment research, audio and video-recordings are collected. These can be used to track the children's progress, but also to perform later treatment fidelity checks in which blinded participants can label, for example, whether the clinician was performing the particular treatment targeted.

\section{Progression of treatment sessions}

Treatment in the Speech Production and Perception Lab typically takes place four times weekly for one hour for four weeks. Time is also scheduled for speech pre-testing (typically, three baselines pre-, a post, a 6-week follow-up and a 6-month follow-up for our design). Some of the measurement sessions include receptive language tests such as the Test for Auditory Comprehension of Language- $3^{\text {rd }}$ Edition (Carrow-Woolfolk, 1998), cognitive tests such as the Kaufman Brief Intelligence Test- $2^{\text {nd }}$ Edition (Kaufman \& Kaufman, 2004), and audiological screening (at $500 \mathrm{~Hz}, 1000 \mathrm{~Hz}, 2000$, and $4000 \mathrm{~Hz}$ at $20 \mathrm{~dB}$ ). Mean length of utterance is always gathered from a language sample. Knowledge of treatment research design (e.g., Kennedy, 2005) is essential for designing and interpreting high-quality treatment research.

The progression of tasks for SSIT is criterion based. Each session begins with a discussion of the speech subsystems (Pennington et al., 2006) with reference to the practice phrases. (As the weeks progress, the reminders become shorter and children are expected to play a larger role in the explanations.) In the first sessions, practice coordinating respiration with phonation takes place with sustained vowels until that is mastered. Based on Pennington et al. (2010), the criterion for progression to the next exercise on the hierarchy is $90 \%$ accuracy in maintaining controlled respiration and phonation over the speech segment/utterance. (This can be 
adjusted based on reasonable expectations for the child). Next, spoken language tasks are targeted on the hierarchy from pharases to single words to sentences (when appropriate) to conversation, with the same criterion (e.g., of 90\% accuracy) for progression to the next level. Thus, for the spoken language exercises, trials are considered correct when a child demonstrates controlled respiration and phonation over an entire speech unit. Because a child can progress to the next level within a session, the clinician needs to be prepared with activities to target the next linguistic unit.

For LSVT LOUD, the first half of each session is devoted to three daily tasks: Sustained vowel phonation, maximal pitch range (rising and falling), and functional phrases. The second half of the session is spent on hierarchical tasks, which progress from week to week (unlike SSIT, in which children advance when its criterion is met). The first week focuses on words or phrases, the second week on sentences, and the third week on reading when appropriate. Week 4 addresses conversationational speech. However, when adapting the LSVT LOUD (Ramig \& Fox, 2010) speech hierarchy for a 3 year old, for example, we followed the following sequence across the weeks: Week 1: Words, Week 2: Phrases, Week 3: Short sentences (often repetition of adult sentences), and Week 4: Conversation with language and turn-taking targets modeled on 3-year olds' conversations. (For more details on time spent per activity, please see Boliek and Fox, 2014.)

In children with dysarthria, unlike in most adults we see with dysarthria, some individuals have not reached sentence level yet and are not reading. Thus, the hierarchies can be adapted to culminate in the maximal linguistic unit the child had achieved. Moreover, if the child tires of a particular activity, other (preferred) activities might be resorted to, following the child's lead as needed, even if such activities target a different level. Such flexibility is sometimes needed simply to maintain the rapport with the child and keep the child speaking and using the new strategies. A lab notebook is kept in the Speech Production and Perception Lab at all times for clinicians to document the protocols used in sessions and any deviations that may have occurred. Instructions and reminders

For both treatment approaches, we provide visual information with instructions. In SSIT, we begin with age-appropriate discussion of the subsystems involved in speech production (Pennington et al., 2006). We provide drawings (e.g., figure 1) and show the children how we take a breath in and phonate upon exhalation. Throughout the session, the clinician provides positive reinforcement and reminders, as needed, for appropriate positioning (e.g., straight back, head slightly tucked), deep breaths, appropriate posture, a clear voice, clear speech, and to monitor phrasing. We use tapping and sometimes metronomes (also available as free iPhone applications, e.g., SilverDial ${ }^{1}$ ) to discuss phrasing of speech, including regulating loudness, controlling rate of speech and syllables per breath, and marking stress. A stimulus cue developed and piloted during the first sessions is also provided for each child to be prompted for his or her new speech skills.

\footnotetext{
${ }^{1}$ This article includes the names of toys, games, and applications that are or may be proprietary terms or trademarks. Their inclusion does not imply that they have acquired a non-proprietary or general significance or any other judgment concerning legal status.
} 

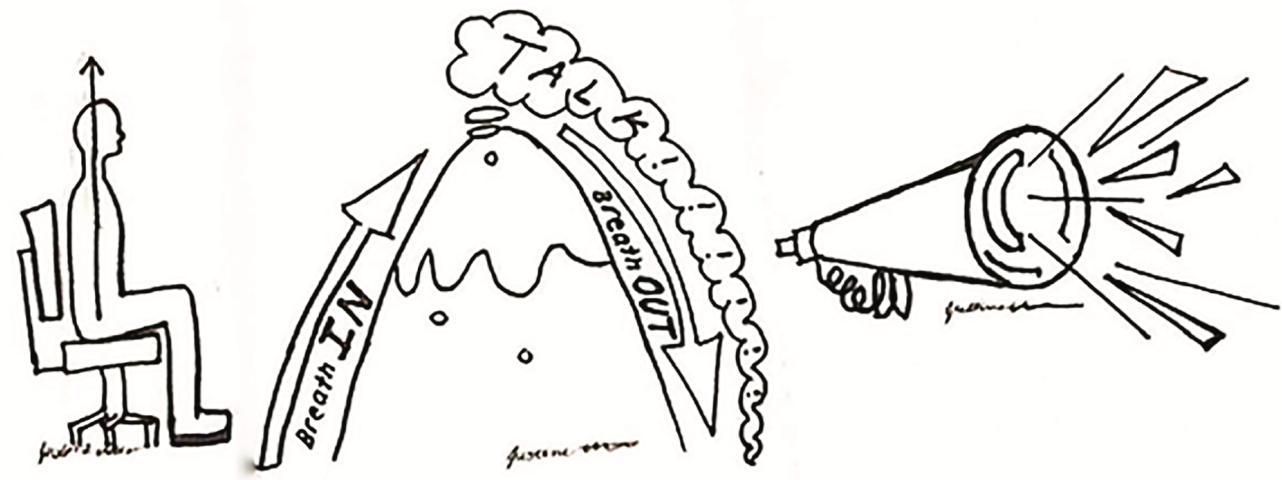

Figure 1. Visual reminder (C) 2014 Justine Allen) for SSIT

Visual reminders are also utilized for LSVT LOUD (see figure 2), but the discussions are minimal. Modeling, "do what I do", and "loud" or "big girl/boy voice" or terminology chosen by the child are used to elicit and maintain appropriate loudness and thereby also target voice quality and intelligibility. A toy microphone can serve as a reminder to encourage louder speech. An iPhone sound level meter (e.g., "dB volume", a free application) or any sound level meter providing visible, concrete feedback is helpful in informing children of how loudly they are speaking and for encouraging them to "speak loud".

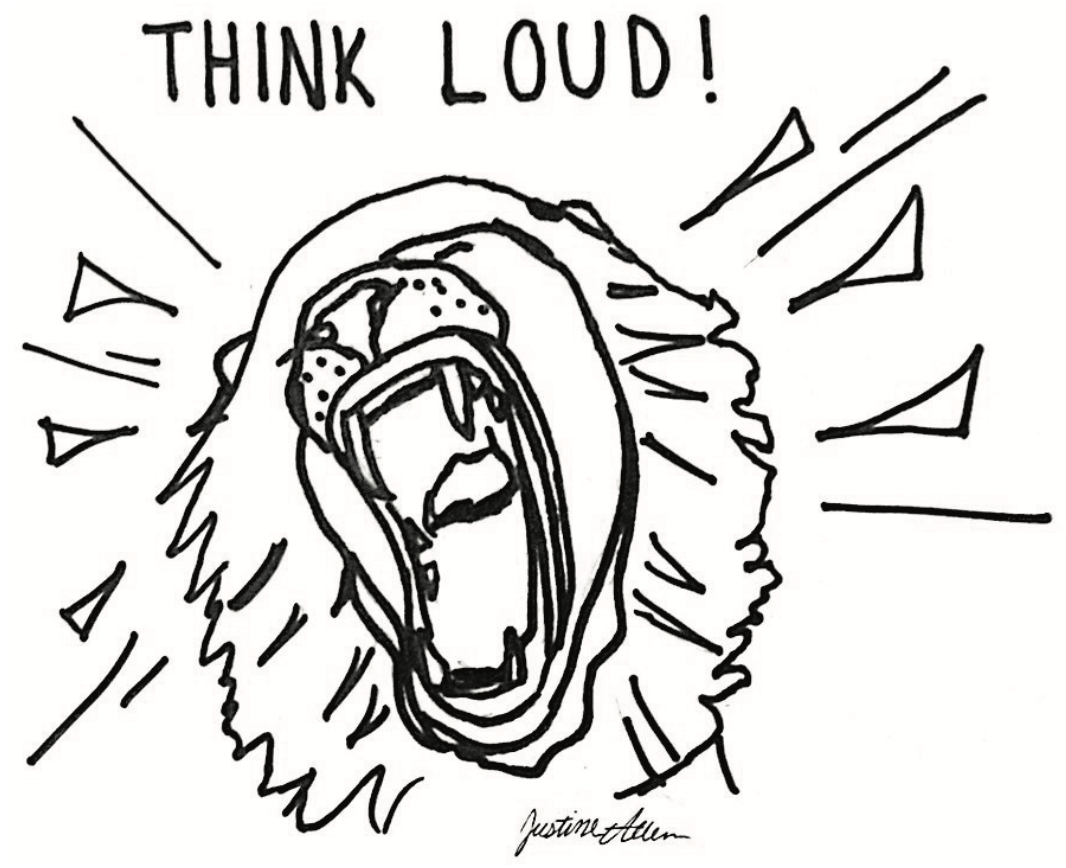

Figure 2. Visual reminder (C) 2014 Justine Allen) for LSVT LOUD

\section{Motivating the child for motor learning}

Both treatment approaches adhere to the principles of motor learning. SSIT utilizes highintensity practice, as well as random practice of target behaviours within and then across 
activities. Based on motor learning principles, feedback is initially provided frequently to promote skill acquisition. Over time, feedback is faded in order to encourage skill retention. Knowledge of performance and of results is incorporated (Pennington et al., 2010). Similarly, LSVT LOUD incorporates motor learning principles in its intensive, high-effort treatment involving repetitive practice, homework, and carry-over assignments. Principles promoting neural plasticity are also incorporated: intensity of practice, saliency of treatment tasks, intervention timing, and complexity of practice (Fox et al., 2006).

Adhering to principles of motor learning is key to any speech treatment (Strand, 1992). Large numbers of practice trials are needed to make gains. One of the main challenges to working with children is maintaining their engagement in repetitive activities long enough and frequently enough to make gains. Treatment research, particularly research adapted from adult studies, does not prepare research clinicians for this aspect of treatment. In table 1 we offer techniques we have used to facilitate the child's completion of large numbers of trials with motivation and often enjoyment. (See also Boliek and Fox, 2014, regarding motivation and rewards.) This table provides a daily breakdown of activities that can be used to motivate children in treatment research. These examples target the interests of a 13-year-old girl receiving SSIT and a 7-year-old boy receiving and LSVT LOUD. Primary motivation and instruction come from positive verbal reinforcement and attention to self-awareness (e.g., for LSVT LOUD, "Wow-did you hear your big-girl voice? I understood exactly what you said!'”).

Table 1: Daily breakdown of motivational activities for SSIT and LSVT LOUD

\begin{tabular}{|c|c|c|}
\hline Day & $\begin{array}{l}\text { SSIT } \\
\text { day-by-day (for a 13-year-old girl) }\end{array}$ & $\begin{array}{l}\text { LSVT LOUD } \\
\text { day-by-day (for a 7-year-old boy) }\end{array}$ \\
\hline 1 & $\begin{array}{l}\text { Sustained vowels } \\
\text { Practice phrases } \\
\text { - Jenga } \\
\text { - Card game (Coconuts), } 4 \\
\text { phrases between turns }\end{array}$ & $\begin{array}{l}\text { Daily tasks } \\
\bullet \quad \text { Jenga } \\
\text { Words/phrases } \\
\bullet \quad \text { Connect Four }\end{array}$ \\
\hline 2 & $\begin{array}{l}\text { Practice phrases: } \\
\text { - Drill style, child chooses a } \\
\text { sentence from a cup and reads } \\
\text { 3x each } \\
\text { - Jenga } \\
\text { Single words } \\
\text { - "Getting to know you" game } \\
\text { - UNO, drill style } \\
\text { - Pictionary }\end{array}$ & $\begin{array}{l}\text { Daily tasks } \\
\bullet \quad \text { Connect Four } \\
\text { Words/phrases } \\
\qquad \quad \text { Jenga (During game, clinician } \\
\text { asks child questions with one- } \\
\quad \text { word responses) }\end{array}$ \\
\hline 3 & $\begin{array}{l}\text { Practice phrases } \\
\text { Single words } \\
\text { - "I'm going on a trip" game } \\
\text { (Clinician and child take turns } \\
\text { naming one item from each }\end{array}$ & $\begin{array}{l}\text { Daily tasks } \\
\qquad \quad \text { Trouble } \\
\text { Words/phrases } \\
\text { - Superhero Bingo }\end{array}$ \\
\hline
\end{tabular}




\begin{tabular}{|c|c|c|}
\hline & $\begin{array}{l}\text { letter of the alphabet to bring on } \\
\text { a trip) } \\
\text { - Pictionary } \\
\text { - Go Fish, drill style }\end{array}$ & $\begin{array}{l}\text { One-Word Story (Clinician and } \\
\text { child take turns saying } \\
\text { consecutive words to make a } \\
\text { story) }\end{array}$ \\
\hline \multirow[t]{5}{*}{4} & Practice phrases & Daily tasks \\
\hline & Single words & - Honey Bee Tree \\
\hline & - Connect Four & \\
\hline & - Mad Libs & Words/phrases \\
\hline & - Sorry! & - Battleship \\
\hline \multirow[t]{5}{*}{5} & Practice phrases & Daily tasks \\
\hline & Single words & - Don't Break the Ice \\
\hline & - UNO, drill style & Sentences \\
\hline & Sentences & $\begin{array}{l}\text { Sentences } \\
\qquad \text { Guess Who? }\end{array}$ \\
\hline & - Hedbanz & - I Spy \\
\hline \multirow[t]{6}{*}{6} & Practice phrases & Daily tasks \\
\hline & Sentences & - Honey Bee Tree \\
\hline & - Would You Rather & \\
\hline & - Guess Who? & Sentences \\
\hline & - iPad barrier game (Child gives & - Don't Spill the Beans \\
\hline & $\begin{array}{l}\text { clinician directions to create a } \\
\text { product) }\end{array}$ & $\begin{array}{l}\text { - Connect Four } \\
\text { - Go Fish }\end{array}$ \\
\hline \multirow[t]{5}{*}{7} & Practice phrases & Daily tasks \\
\hline & Sentences & - Space Faces game \\
\hline & - iPad barrier game & \\
\hline & - Hedbanz & Sentences \\
\hline & - Would You Rather & - Go Fish \\
\hline \multirow[t]{5}{*}{8} & Practice phrases & Daily tasks \\
\hline & Sentences & - Space Faces \\
\hline & - Hedbanz & \\
\hline & - Guess Who? & Sentences \\
\hline & & $\begin{array}{l}\text { - Guess Who? } \\
\text { - Trouble }\end{array}$ \\
\hline \multirow[t]{5}{*}{9} & Practice phrases & Daily tasks \\
\hline & Sentences & - Connect Four \\
\hline & - Two Truths and a Lie & - Bowling \\
\hline & & Reading \\
\hline & & $\begin{array}{l}\text { - Cloudy with a Chance of } \\
\text { Meatballs (Barrett, 1978) }\end{array}$ \\
\hline \multirow[t]{3}{*}{10} & Practice phrases & Daily tasks \\
\hline & Sentences & - War card game \\
\hline & $\begin{array}{l}\text { - iPad barrier game } \\
\text { - UNO, drill style }\end{array}$ & Reading \\
\hline
\end{tabular}




\begin{tabular}{|c|c|c|}
\hline & & $\begin{array}{l}\text { In the Trees, Honey Bees } \\
\text { (Mortensen, 2009) }\end{array}$ \\
\hline 11 & $\begin{array}{l}\text { Practice phrases } \\
\text { Sentences } \\
\text { - UNO } \\
\text { - iPad barrier game } \\
\text { - LIFE game (Child reads all } \\
\text { sentences from game cards and } \\
\text { uses full sentences during } \\
\text { discourse) }\end{array}$ & $\begin{array}{l}\text { Daily tasks } \\
\text { - Crazy Eights } \\
\text { Reading } \\
\text { - Swirl by Swirl: Spirals in } \\
\text { Nature (Sidman, 2011) } \\
\text { - The Foot Book: Dr. Seuss's } \\
\text { Wacky Book of Opposites } \\
\text { (Seuss, 1996) }\end{array}$ \\
\hline 12 & $\begin{array}{l}\text { Practice phrases } \\
\text { Sentences } \\
\text { - Twenty Questions } \\
\text { - Rory's Story Cubes (Using } \\
\text { cubes with items depicted on } \\
\text { them, child and clinician take } \\
\text { turns narrating a story) } \\
\text { - Taboo game }\end{array}$ & $\begin{array}{l}\text { Daily tasks } \\
\text { - Hoot, Owl, Hoot board game } \\
\text { - Life on Earth Bingo } \\
\text { Reading } \\
\qquad \quad \text { Turtle, Turtle, Watch Out! } \\
\quad \text { (Sayre, 2010) }\end{array}$ \\
\hline 13 & $\begin{array}{l}\text { Practice phrases } \\
\text { Conversation } \\
\text { - Discussion of fourth of July } \\
\text { weekend } \\
\text { - LIFE game }\end{array}$ & $\begin{array}{l}\text { Daily tasks } \\
\text { - War card game } \\
\text { Conversation } \\
\text { - Topic cards from Topic Talk } \\
\quad \text { game }\end{array}$ \\
\hline 14 & $\begin{array}{l}\text { Practice phrases } \\
\text { Conversation } \\
\text { - Conversation regarding day's } \\
\text { activities at camp } \\
\text { - Rory's Story Cubes } \\
\end{array}$ & $\begin{array}{l}\text { Daily tasks } \\
\text { - Hoot, Owl, Hoot board game } \\
\text { Conversation } \\
\bullet \quad \text { Rory's Story Cubes }\end{array}$ \\
\hline 15 & $\begin{array}{l}\text { Practice phrases } \\
\text { Conversation } \\
\text { - Rory's Story Cubes } \\
\text { - Topic cards from Topic Talk } \\
\text { game }\end{array}$ & $\begin{array}{l}\text { Daily tasks } \\
\text { - Space Faces } \\
\text { Conversation } \\
\text { - Topic cards from Topic Talk } \\
\quad \text { game } \\
\end{array}$ \\
\hline 16 & $\begin{array}{l}\text { Practice phrases } \\
\text { Conversation } \\
\text { - Making ice cream sundaes } \\
\text { (Child gives clinician multiple- } \\
\text { step directions to create } \\
\text { identical sundaes) } \\
\text { - Discussion regarding plans for } \\
\text { the rest of the summer vacation }\end{array}$ & $\begin{array}{l}\text { Daily tasks } \\
\text { - } \text { Bowling } \\
\text { Conversation } \\
\text { - Discussion regarding camp and } \\
\text { rest of summer vacation } \\
\text { - Practice with mock } \\
\text { conversations with friends in } \\
\text { new school year }\end{array}$ \\
\hline
\end{tabular}


Motivation can come through communication-based motivational games catered to the child's interests. Asking a young child to read or repeat the same phrases multiple times (as is often done with adults with dysarthria) can lead to tears. Instead, a game of Jenga, for example, can be used to render the activity more enjoyable. Each phrase can be written on paper and pasted onto a Jenga block or written directly onto the block in erasable ink. The child and clinician take turns removing a Jenga block. For each block removed, the child says the phrase (either by reading or repeating after the clinician.) For a child with fine motor difficulties, the clinician assists with the manual task.

At the sentence level, barrier games can be used to target intelligibility (and comprehensibility) directly. For example, if both the clinician and child have an iPad (or a paper and markers), the child can design a cupcake using "Easy Bake Oven" (or drawing a cupcake). The child then utters instructions such as "Make a chocolate cupcake and add white ice cream and rainbow sprinkles." At the end, the child and clinician can compare their final products (e.g., a chocolate cupcake with red icing and yellow sprinkles) and assess, usually with laughter, whether the message was accurately transmitted. Repairs, if needed, can be made.

As clinicians aim to maximize the children's motor output throughout the session, they must be mindful that some children require extensive time for certain games, especially those involving tasks that are more cognitively challenging, and may decrease their motor output when playing certain games. Clinicians then switch to faster-paced games (e.g., Connect Four) generating more speech. Clinicians must also be aware of their own speech output, ensuring that they allow adequate practice for the child.

Homework and carryover

Homework and carry-over after the treatment program ends are discussed with the parents in advance and their importance for maintenance of gains is emphasized. We ask parents to practice facilitating the children's homework in our presence so that we can provide input. We send home reminders of the cues for better speech habits (e.g., a drawing of appropriate posture and breathing, and clear voice for SSIT or with the cue "LOUD" for LSVT LOUD [see figures 1 and 2]). Checklists are also provided to the children or parents for them to indicate when the children have practiced. For SSIT, children are asked to spend 10-15 minutes daily using the strategies during a specific activity (e.g., over dinner, speaking with siblings, on his or her drive home). We ask them to video call (e.g., using Facetime) clinicians every other day to demonstrate their strategies in use. For LSVT LOUD, the homework regimen is prescribed (see Boliek and Fox, 2014, for details). For both treatment approaches, customized activities are sent home so that, for example, children can practice naming their favorite characters in a comic strip series or movie or read age-appropriate books.

It should be noted that research has shown mixed results for maintenance of skills after treatment has ended. For example Pennington et al. (2006) found that intelligibility scores returned to pre-treatment levels 7 weeks after treatment for all but one child. However, in Pennington et al. (2010; 2013), levels were maintained 6 to 12 weeks post-treatment.

Maintenance of improvements in Fox and Boliek (2012) varied among the children. Thus, further research is needed to understand the dosage, motivation, and monitoring of homework required for children's gains to be maximized during treatment and maintained or increased thereafter.

\section{Specifics of outcome measurement}

To document the presence or absence of changes as a function of treatment in speech treatment research, it is essential for measurement to yield valid and reliable findings. It is thus crucial for treatment researchers to gather high-quality audio recordings, free from extraneous noise- a challenge, given children's active natures and the movement disorders often 
accompanying motor speech disorders. Furthermore, the sound pressure level of the signal requires precise measurement. Small changes in the mouth-to-microphone distance or in the input setting can affect audibility and thus intelligibility.

The outcome measurement protocol in the Speech Production and Perception Lab incorporates a hierarchy of speech tasks. Single-word tasks include the AAPS-3 (Fudala, 2001) and repetition of real words and nonsense words produced by a native speaker of American English (AE), presented via loudspeakers on a computer. Sentence-level tasks include repetition of sentences and functional phrases (or practice phrases). Conversational level tasks include a picture-description task, a language sample (using a child-centred topic, such as describing a typical day at school), and the retelling of a short wordless video from YouTube. Other tools frequently used for assessing the speech of children with dysarthria include the Test of Children's Speech Plus (TOCS+) software program (Hodge \& Daniels, 2007) and the Children's Speech Intelligibility Measures (Wilcox \& Morris, 1999).

Recording children's speech

We record children's speech in a sound-treated booth at Teachers College. (Clinicians treating the children are not present during post-testing because their presence may cue the children to produce "treatment" speech and thus affect the results.) The children are seated as they usually sit, with no instructions provided on posture. A microphone is placed $8 \mathrm{~cm}$ from the child's upper lip. Stand-alone microphones are not used in the Speech Production and Perception Lab for treatment studies because children's distances from this type of microphone vary as they move. Instead we have used a head-mounted microphone, and more recently, an omnidirectional lavalier microphone (Countryman EMW) taped to the children's foreheads (Fox \& Boliek, 2012). The lavalier microphone system has produced the most noise-free recordings and the least discomfort for the children. Headbands maintain the microphone in place - the children respond well to being told they look like rock stars! The signal passes from the microphone via a Shure (Prologue 200M) mixer to a sound card (Turtle Beach Riviera) of a desktop computer (Dell Pentium 4) by means of Soundforge (Sony Creative Software) software. The sample rate is $22,050 \mathrm{~Hz}$ (although 44,100 can also be used), with 16-bit resolution and is on a mono channel.

Calibration is essential for certification of the actual sound pressure level of the original signal and the ability to preserve relative differences in sound pressure level among speech samples, as well as to measure changes in sound pressure level as a function of treatment. In the Speech Production and Perception Lab, calibration involves generating a tone on a music tuner (KORG LCA-120 Chromatic) placed adjacent to the microphone. As many children have short attention spans and low tolerance for tuner tones, we calibrate before and after testing sessions using a Styrofoam head as a model, rather than calibrating with the microphones on the children.

Calibration set-up (see figure 3) involves a Styrofoam head with a lavalier microphone mounted at the forehead, a music tuner positioned at the place of the mouth (representing the child's mouth), and a (Galaxy-Audio CM-140) sound level meter (SLM) placed adjacent to the microphone (also $8 \mathrm{~cm}$ away from the tuner). The experimenter plays a tone on the tuner and notes the sound pressure level on a SLM placed at the same distance at the beginning and at the end of each session for confirmation and for subsequent restoration to the original sound pressure level. 


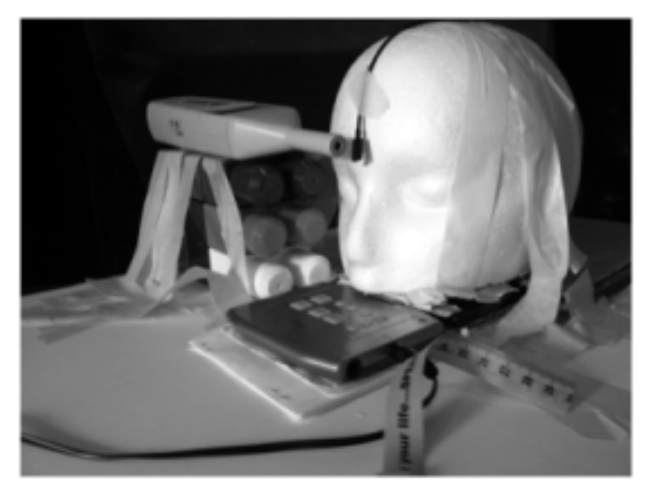

Figure 3. Calibration unit

For recording, we set the input at a low level at which a high-quality signal is recorded, but is not distorted at child's peak amplitude (allowing for an sound pressure level increase) and then do not change the input settings after calibration for the entire study. Alternatively, children can be recorded at the optimal input levels for their speech (i.e., the highest level without distortion). During analysis, the difference between the sound pressure level of the calibration tone noted on the sound level meter and the sound pressure level on the recording is applied to the sound pressure level on the recording (by means of the Praat software program [Boersma \& Weenink, 2013]) to restore the sound pressure level to its actual value.

For playback to listeners, the researcher adjusts the volume knob on a loudspeaker to replay the recorded tone at the original sound pressure level (as measured on a sound level meter $8 \mathrm{~cm}$ away). Alternatively, some researchers set the output sound pressure level at a comfortable listening level (e.g., peaking at $70 \mathrm{~dB}$ sound pressure level $50 \mathrm{~cm}$ away from loudspeaker). As a result of careful calibration, the relative sound pressure level differences among speakers and conditions are maintained.

Regardless of the recording or calibration system used, it is essential to record several tokens of each utterance to the extent possible. Many tokens will need to be discarded due to noise, children's whimsical behaviour, and the difficulty recording and measuring whispered and otherwise atypical speech, even with the finest recording systems.

\section{Specifics of acoustic analysis}

Acoustic analysis is an informative means by which treatment researchers can measure treatment-related changes in speech production in children with dysarthria. Vowels are of particular interest because they are important for intelligibility in typical speech (Bradlow, Torretta, \& Pisoni, 1996; Kewley-Port, Burkle, \& Lee, 2007) and in individuals with dysarthria (Ansel \& Kent, 1992; Higgins \& Hodge, 2002; Lee \& Hustad, 2013). First formant (F1) frequencies relate (inversely) to tongue height, whereas second formant frequencies (F2) provide information on the front-back dimension of the highest part of the tongue (Raphael, Borden, \& Harris, 2011). Both formants lower with age until the children are approximately age 18 years (Lee \& Hustad, 2013). Children with dysarthria tend to have smaller vowel spaces than typicallydeveloping children, but a robust relationship between an expanding vowel space and greater intelligibility has been shown consistently in the literature (Higgins \& Hodge, 2002; Lee \& Hustad, 2013). Vowel space expansion has been documented following LSVT LOUD for adults with dysarthria due to Parkinson Disease (Sapir et al., 2007). However, less is known about whether children's vowel space expands after speech treatment.

We examine children's repetition of nonsense words (among other measures) before and 
after speech treatment. Nonsense words are used in order to glean information about vowel production without the influence of lexical effects (Neuman \& Hochberg, 1983). This also readily permits the targeted speech sounds to be produced in consistent phonological contexts and thus not be influenced differentially by coarticulation (Hillenbrand, Clark, \& Nearey, 2001; Levy, 2009).

Below we provide results yielded by such analysis, with a focus on spectral analysis of vowels. For acoustic analysis in the Speech Production and Perception Lab we generally use Praat (Boersma \& Weenink, 2013) software, which can be downloaded at http://www.fon.hum.uva.nl/praat/. This is cost-free and relatively user-friendly software. (For more extensive acoustic analysis, MATLAB [Mathworks, Inc.] systems can be programmed.) A manual for using Praat can be found at http://savethevowels.org/praat/UsingPraatforLinguisticResearchLatest.pdf.

\section{Example of acoustic analysis}

We examined the acoustics of the vowels produced by the three children with dysarthria due to CP (P1, P2, and P3) whose speech function was studied in Levy et al. (2012). The children's speech was recorded using the techniques described above (in this case a Shure [SM10A] unidirectional headset microphone). For the present investigation of their production of vowels in nonsense words pre- and immediately post-treatment, the children were asked to repeat the pre-recorded utterance /hVba/ with the AE monophthongs $(/ \mathrm{i} /, / \mathrm{I} /, / \varepsilon /, / æ /, / \mathrm{N} /, / \mathrm{u} /, / \mathrm{J} /, / \mathrm{a} /)$ produced by an adult native speaker of AE from the New York regional area. Acoustic analysis of the vowel midpoints was performed by means of Praat (Boersma \& Weenink, 2013) by the author and a research assistant. Reliability was $81 \%$. When discrepancies arose, the vowels were reanalysed and final values were determined by consensus.

Figures 4, 5, and 6 represent the F1 (y axis) and F2 (x axis) frequencies of the vowels produced by the three children with dysarthria. The solid circles represent pre-treatment productions and the striped triangles represent post-treatment productions. Ellipses (created with the "shape" tool in Microsoft Excel) surround a pre- and a post-treatment production of each vowel. (Baseline productions were relatively consistent for P1 and P2, but not for P3, the child with dysarthria and apraxia.) Figure 4 reveals acoustic vowel space expansion for P1 (the 8;8 year old with mild dysarthria). After LSVT LOUD, most vowels were represented more peripherally in acoustic vowel space than pre-treatment. In addition, as indicated by the F1 and F2 pre-treatment vs. post-treatment differences, low vowels were produced with a lower tongue position post-treatment and high back vowel /u/ was produced with the tongue higher in the oral cavity post-treatment.

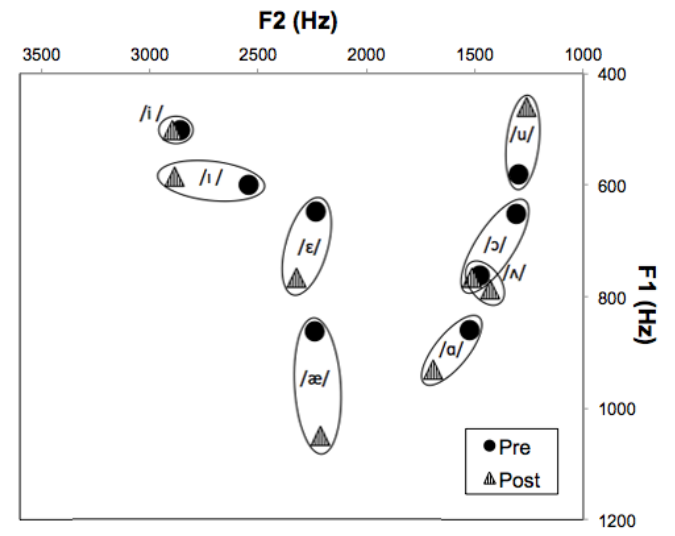

Figure 4. P1 (8;8 year old with dysarthria) vowels in nonsense words pre- and post-LSVT LOUD 


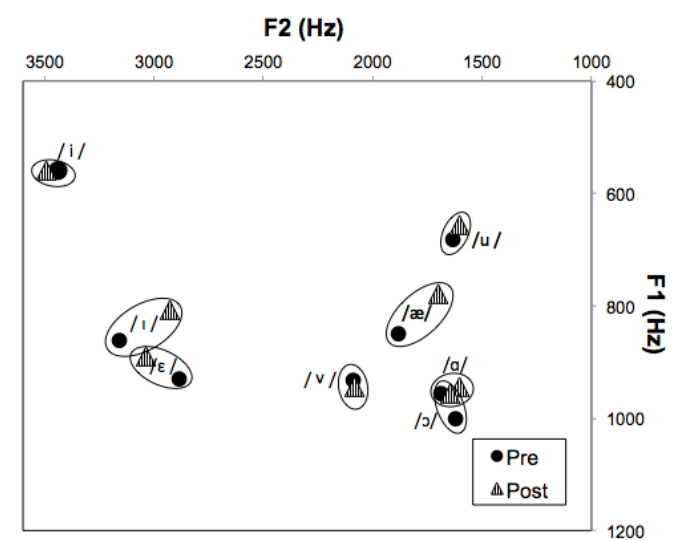

Figure 5. P2 (3;3 year old with dysarthria) vowels in nonsense words pre- and post-LSVT LOUD

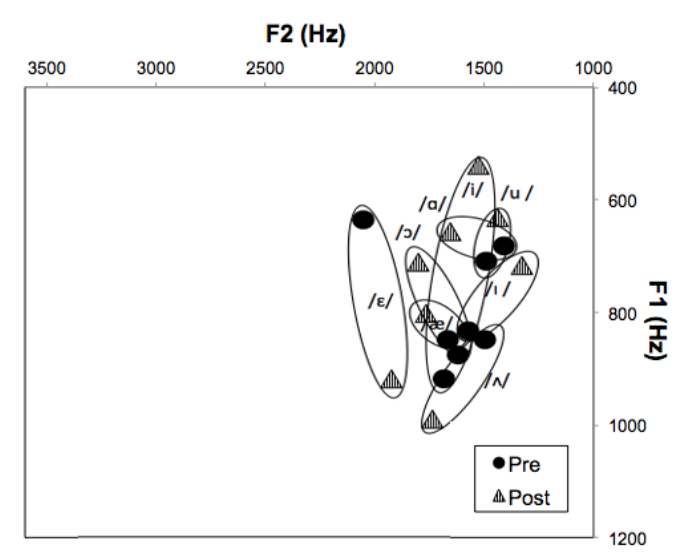

Figure 6. P3 (9;6 year old with dysarthria and apraxia) vowels in nonsense words pre- and postSSIT

Vowel area in Hz was calculated (on Excel) using the formula of the area of an irregular quadrilateral (Vorperian \& Kent, 2007): Area $=.5 *\{(/ \mathrm{i} / \mathrm{F} 2 * / æ / \mathrm{F} 1+/ æ / \mathrm{F} 2 * / \mathrm{a} / \mathrm{F} 1+/ \mathrm{a} / \mathrm{F} 2 * / \mathrm{u} / \mathrm{F} 1$ $+/ \mathrm{u} / \mathrm{F} 2 * / \mathrm{i} / \mathrm{F} 1)-(/ \mathrm{i} / \mathrm{F} 1 * / \mathfrak{x} / \mathrm{F} 2 /+/ \mathfrak{m} / \mathrm{F} 1 * / \mathrm{a} / \mathrm{F} 2+/ \mathrm{a} / \mathrm{F} 1 * \mathrm{uF} 2+/ \mathrm{u} / \mathrm{F} 1 / * / \mathrm{i} / \mathrm{F} 2)\}$. (See table 2.) These calculations confirmed that vowel space expanded by $202,535 \mathrm{~Hz}$ for P1.

Table 2: Vowel area (in $\mathrm{Hz}$ ) at pre-treatment, post-treatment, and difference between pre- and post-treatment

\begin{tabular}{cccc} 
Participant & Pre (Hz) & Post $\mathbf{( H z )}$ & Difference $(\mathbf{H z})$ \\
\hline P1 & 354219 & 556754 & 202535 \\
P2 & 194071 & 133398 & -60673 \\
P3 & -1657 & 8626 & 10282 \\
\hline
\end{tabular}


For $\mathrm{P} 2$, the 3;3 year old with moderate dysarthria, acoustic vowel space was more constricted following treatment (figure 5). Although the slightly lower F2 suggests that after LSVT LOUD, some back vowels were produced with a slightly more retracted tongue, her vowels were generally characterized by a reduced F1, reflecting a higher tongue position, posttreatment. Differences in her front vowel production following treatment were inconsistent. This young child typically repeated the target front mid-low vowel /æ/ as low back vowel /a/ both preand post-treatment. The vowel area calculation (Voperian \& Kent, 2007) confirmed that her vowel space was reduced $(-60673 \mathrm{~Hz})$ following treatment (table 2).

As indicated by the formant values depicted in figure 6, vowels produced by P3 (age 9.6, with moderate dysarthria and severe apraxia) revealed a highly restricted, centralized acoustic vowel space and inaccurate production before and after SSIT. Following treatment, her vowel space shifted in variable directions. This child's acoustic vowel space pre-treatment had a negative value because of the inaccuracies of her vowel production. For example, the target high front vowel /i/ was produced as a low back vowel before treatment. Calculations (Voperian \& Kent, 2007) revealed vowel space expansion $(10282 \mathrm{~Hz}$ ) for this child following SSIT (table 2). Perhaps of more consequence than the vowel space expansion for this child, after treatment, although her vowels were still predominantly inaccurately produced, certain vowels approximated more closely their target values. For example, target high front $/ \mathrm{i}$ / was produced as a higher, albeit still back, vowel, thus contributing to a less restricted vowel space (and greater articulatory precision [Levy et al., 2012]) post-treatment.

In summary, two out of the three children (P1 and P3) revealed greater acoustic vowel space following (LSVT LOUD and SSIT). However, for all three children, Levy et al. (2012) found increased articulatory accuracy (at word- and conversational-speech levels) according to the AAPS-3 (Fudala, 2001), as well as post-treatment stimuli preferred and judged more intelligible than pre-treatment stimuli. Thus, for both children whose acoustic vowel space expanded, the present findings are consistent with findings in Levy et al. (2012), as with the vowel space expansion found following treatment on adults with dysarthria (Sapir et al., 2007). The 3;3 year old child (P2), whose vowel space contracted following treatment, presented with phonological processes lingering beyond age expectations. However, she did not show vowel accuracy deficits on the AAPS-3 (Fudala, 2001) before or after treatment, other than ageappropriate errors on r-coloured vowels. In her case, vowel space constriction did not result in reduced intelligibility. More accurate consonant production or prosodic changes (including 9$16 \mathrm{~dB}$ sound pressure level increases in sound pressure level), rather than changes in vowel production following treatment likely contributed to increases in articulatory accuracy and perceived intelligibility, as well as listeners' preference for her post-treatment speech. Listener intelligibility judgments and preference for the child's post-treatment speech may also have been influenced by the child's greater confidence following treatment, a phenomenon suggested by Pennington et al. (2013), who found no relationship between the gains in communicative participation in children with dysarthria and their increases in speech intelligibility following treatment.

As there are individuals who reveal stronger treatment responses (Boliek \& Fox, 2014), there may also be individuals whose acoustic vowel space increases more than others' as a function of treatment. Children's age, vocal tract anatomy, type and degree of motor deficit, phonological development, individual characteristics, and growth spurts (Vorperian \& Kent, 2007) are likely contributing factors. Further research is needed to explore the relationships among speech treatment, expansion of vowel space, intelligibility, and communicative participation by children with CP. More objective measures of intelligibility, such as percent 
vowels accurately transcribed orthographically (Hustad, 2006), are underway in the Speech Production and Perception Lab. Results suggest that special attention should be paid to treating children's front and low vowel productions, as these are the least intelligible vowels for most of our participants (Levy, Seid, Chen, Leone, Moya-Gale, Hsu, \& Ramig, 2014).

\section{Conclusion}

Researchers and clinicians encounter a multitude of challenges as they treat children with dysarthria. However, well-planned strategies for motivating the children during treatment, as well as high quality recording and analysis techniques, render this research less daunting and more rewarding for all involved. It is hoped that continued provision of specifics regarding dysarthria treatment research techniques will further clinical research on this topic with the goal of generating effective treatments that will help the children communicate and socialize with greater ease and success.

\section{Acknowledgments}

Many thanks to the children and their families, and to Kellianne Beers, Carol Boliek, Stephen Camarata, Wenli Chen, Cynthia Fox, Andrew Gordon, Sih-Chiao Hsu, Binna Lee, Lauren Liria, Gemma Moya-Gale, Lorraine Ramig, Elanna Seid, Jennifer Spielman, and Kenay Sudler. The author also thanks Justine Allen for her drawings, which are used with her permission as figures 1 and 2. Thanks also to Katie Mosher and Katrina Andres, the main sources for the innovative, motivating games. 


\section{References}

Ansel, B. M., \& Kent, R. D. (1992). Acoustic-phonetic contrasts and intelligibility in the dysarthria associated with mixed cerebral palsy. Journal of Speech and Hearing Research, 35, 296-308.

Barrett, J. (1978). Cloudy with a chance of meatballs. New York, NY: Simon \& Schuster.

Boersma, P., \& Weenink, D. (2013). Praat: doing phonetics by computer (Version 4.3.01)

[Computer program]. Version 5.3.53, retrieved from http://www.praat.org/

Boliek, C. A., \& Fox, C. M. (2014). Individual and environmental contributions to treatment outcomes following a neuroplasticity-principled speech treatment (LSVT LOUD) in children with dysarthria secondary to Cerebral Palsy. International Journal of SpeechLanguage Pathology.

Bradlow, A. R., Torretta, G. M., \& Pisoni, D. B. (1996). Intelligibility of normal speech I: Global and fine-grained acoustic-phonetic talker characteristics. Speech Communication, 20, 255272.

Butler, C., \& Darrah, J. (2001). AACPDM Evidence report: Effects of neurodevelopmental treatment (NDT) for cerebral palsy. Developmental Medicine and Child Neurology, 43(11), 778-790.

Carrow-Woolfrock, E. (1998). Test for Auditory Comprehension of Language (3 ${ }^{\text {rd }}$ ed.) [TACL-3]. Austin, TX: Pro-Ed.

Dr. Seuss. (1996). The foot book: Dr. Seuss's wacky book of opposites. New York, NY: Random House. (Original work published 1968).

Fox, C. M. \& Boliek, C. A. (2012). Intensive voice treatment (LSVT LOUD) for children with spastic cerebral palsy and dysarthria. Journal of Speech, Language, and Hearing Research, 55, 930-945.

Fox, C. M., Ramig, L. O., Ciucci, M. R., Sapir, S., McFarland, D. H., \& Farley, B. G. (2006) The science and practice of LSVT/LOUD: neural plasticity-principled approach to treating individuals with Parkinson disease and other neurological disorders. Seminar of Speech Language, 27(4), 283-299.

Fudala, J. B. (2001). Arizona Articulation Proficiency Scale (3 ${ }^{\text {rd }}$ ed.) (AAPS-3). Los Angeles, CA: Western Psychological Services.

Goffman, L. (2004). Kinematic differentiation of prosodic categories in normal and disordered language development. Journal of Speech, Language, and Hearing Research, 47, 10881102.

Green, J. R., Moore, C. A., \& Reilly, K. J. (2002). The sequential development of jaw and lip control for speech. Journal of Speech, Language, and Hearing Research, 45, 66-79.

Higgins, C. M., \& Hodge, M. M. (2002). Vowel area and intelligibility in children with and without dysarthria. Journal of Medical Speech Language Pathology, 10, 271-278.

Hillenbrand, J. M, Clark, M. J., \& Nearey, T. M. (2001). Effects of consonant environment on vowel formant pattern. Journal of the Acoustical Society of America, 109, 748-763.

Hodge, M. \& Daniels, J. (2007). Test of Children's Speech Plus (TOCS+ Plus) ver. 5.3. Edmonton, AB: University of Alberta [computer program].

Hodge, M. M. \& Wellman, L. (1999). Management of children with dysarthria. In A. J. Caruso, \& E. A. Strand (Eds.), Clinical management of motor speech disorders in children. New York, NY: Thieme.

Hustad, K. C. (2006). Estimating the intelligibility of speakers with dysarthria. Folia Phoniatrica et Logopaedica, 58, 217-228. 
Hustad, K. C., Gorton, K., \& Lee, J. (2010). Classification of speech and language profiles in 4year-old with cerebral palsy: A prospective preliminary study. Journal of Speech,

Language, and Hearing Research, 53, 1496-1513.

Kaufman, A. S., \& Kaufman, N. L. (2004). Kaufman Brief Intelligence Test (2 ${ }^{\text {nd }}$ ed.) [KBIT-2]. San Antonio, TX: Pearson.

Kennedy, C. H. (2005). Single-case designs for educational research. Boston, MA: Allyn and Bacon.

Kent, R. D. (2000). Research on speech motor control and its disorders: A review and prospective. Journal of the Communication Disorders, 33, 391-428.

Kewley-Port, D., Burkle, T. Z., \& Lee, J. H. (2007). Contribution of consonant versus vowel information to sentence intelligibility for young normal-hearing and elderly hearingimpaired listeners. Journal of the Acoustical Society of America, 122, 2365-2375.

Lee, J., \& Hustad, K. C. (2013). A preliminary investigation of longitudinal changes in speech production over 18 months in young children with cerebral palsy. Folia Phoniatrica et Logopedica, 65(1), 32-39.

Levy, E. S. (2009). Language experience and consonantal context effects on perceptual assimilation of French vowels by American-English learners of French. Journal of the Acoustical Society of America, 125, 1138-1152.

Levy, E. S., Ramig, L. O., \& Camarata, S. M. (2012). The effects of two speech interventions on speech function in pediatric dysarthria. Journal of Medical Speech-Language Pathology, 20(4), 82-87.

Levy, E. S., Seid, E., Chen, W., Leone, D., Moya-Gale, G., Hsu, S.-C., \& Ramig, L. (2014). Vowel intelligibility in children with dysarthria. Poster presented at the Motor Speech Conference, Sarasota, FL.

LSVT Global. (2013). LSVT Global. Retrieved from http://www.lsvtglobal.com/

Mortensen, L. (2009). In the trees, honey bees. Nevada City, CA: Dawn Publications.

Neuman, A. \& Hochberg, I. (1983). Children's perception of speech in reverberation. Journal of the Acoustical Society of America, 73, 2145-2149.

Pennington, L., Miller, N., \& Robson, S. (2009). Speech therapy for children with dysarthria acquired before three years of age. Cochrane Database Systematic Reviews, 4, 1-19.

Pennington L, Miller N, Robson S, \& Steen N. (2010). Intensive speech and language therapy for older children with cerebral palsy: a systems approach. Developmental Medicine and Child Neurology, 52(4), 337-344.

Pennington, L., Roelant, E., Thompson, V., Robson, S., Steen, N., \& Miller, N. (2013). Intensive dysarthria therapy for younger children with cerebral palsy. Developmental Medicine and Child Neurology, 55, 464-471.

Pennington, L., Smallman, C., \& Farrier, F. (2006). Intensive dysarthria therapy for older children with cerebral palsy: findings from six cases. Child Language Teaching and Therapy, 22(3), 255-273.

Ramig, L., \& Fox, C. (2010). LSVT LOUD Training and Certification Workshop Binder. Tucson, AZ: LSVT Global.

Ramig, L., Sapir, S., Fox, C., \& Countryman, S. (2001). Changes in vocal intensity following intensive voice treatment (LSVT) in individuals with Parkinson disease: A comparison with untreated patients and normal age-matched controls. Movement Disorders, 16, 7983.

Raphael, L. J., Borden, G.J., \& Harris, K.S. (2011). Speech science primer: Physiology, acoustics, and perception of speech ( $6^{\text {th }}$ ed.). Philadelphia, PA: Lippincott Williams \& Wilkins. 
Robey, R. R. (2004). A five-phase model for clinical-outcome research. Journal of Communication Disorders, 37, 401-411.

Sapir, S., Spielman, J. L., Ramig, L. O., Story, B. H., \& Fox, C. (2007). Effects of intensive voice treatment (the Lee Silverman Voice Treatment [LSVT]) on vowel articulation in dysarthric individuals with idiopathic Parkinson disease: Acoustic and perceptual findings. Journal of Speech, Language, and Hearing Research, 50(4), 899-912.

Sayre, A. P. (2010). Turtle, turtle, watch out! Watertown, MA: Charlesbridge Publishing.

Sidman, J. (2011). Swirl by swirl: Spirals in nature. New York, NY: Houghton Mifflin Harcourt.

Smith, A. \& Goffman, L. (2004). Interaction of language and motor factors in speech production. In B. Maasen, R.D. Kent, H.F.M. Peters, H. Peters, P. van Lieshout, \& W. Hulstijn (Eds.), Speech motor control in normal and disordered speech. Oxford: Oxford University Press, 225-252.

Strand, E. A. (1992). The integration of speech motor control and language formulation in models of acquisition. In R. S. Chapman (Ed.), Processes in language acquisition and disorders (pp. 86-107). St Louis, MO: Mosby-Year Book.

Strand, E. A. (1995). Treatment of motor speech disorders in children. Seminars in Speech and Language 16, 126-39.

Vorperian, H. K., \& Kent, R. D. (2007). Vowel acoustic space development in children: A synthesis of acoustic and anatomic data. Journal of Speech, Language, and Hearing Research, 50(6), 1510-1545.

Wilcox, K., \& Morris, S. (1999). Children's Speech Intelligibility Measure (CSIM). San Antonio, TX: The Psychological Corporation. 